ORIGINAL ARTICLE

\title{
Should football players wear custom fitted mouthguards? Results from a group randomised controlled trial
}

\author{
C Finch, R Braham, A McIntosh, P McCrory, R Wolfe
}

Injury Prevention 2005;11:242-246. doi: 10.1136/ip.2004.006882

See end of article for authors' affiliations a.r.ers aftiations

Correspondence to: Professor C Finch, NSW Injury Risk Management Research Centre, University of New South Wales, Sydney, NSW, Australia 2052; c.finch@ unsw.edu.au

Accepted 21 March 2005
Objective: Head/orofacial (H/O) injuries are common in Australian rules football. Mouthguards are widely promoted to prevent these injuries, in spite of the lack of formal evidence for their effectiveness. Design: The Australian football injury prevention project was a cluster randomized controlled trial to evaluate the effectiveness of mouthguards for preventing $\mathrm{H} / \mathrm{O}$ injuries in these players.

Setting and subjects: Twenty three teams (301 players) were recruited from the largest community football league in Australia.

Intervention: Teams were randomly allocated to either the MG: custom made mouthguard or C: control (usual mouthguard behaviours) study arm.

Main outcome measures: All injuries, participation in training and games, and mouthguard use were monitored over the 2001 playing season. Injury rates were calculated as the number of injuries per 1000 person hours of playing time. Adjusted incidence rate ratios were obtained from Poisson regression models.

Results: Players in both study arms wore mouthguards, though it is unlikely that many controls wore custom made ones. Wearing rates were higher during games than training. The overall rate of $\mathrm{H} / \mathrm{O}$ injury was 2.7 injuries per 1000 exposure hours. The rate of $\mathrm{H} / \mathrm{O}$ injury was higher during games than training. The adjusted $\mathrm{H} / \mathrm{O}$ injury incidence rate ratio was 0.56 (95\% $\mathrm{Cl} 0.32$ to 0.97$)$ for $\mathrm{MG}$ versus $\mathrm{C}$ during games and training, combined.

Conclusions: There was a significant protective effect of custom made mouthguards, relative to usual mouthguard use, during games. However, the control players still wore mouthguards throughout the majority of games and this could have diluted the effect. n n its 1995 report Football Injuries to the Head and Neck, the National Health and Medical Research Council of Australia identified the prevention of these injuries as requiring urgent attention because they are associated with a risk of permanent brain or spinal damage; cumulative brain damage from repeated injury; reduced scholastic performance; neuropsychological deficits; and cumulative trauma. ${ }^{1}$ This report also identified the development of specific injury countermeasures to prevent these injuries as a research priority.

Mouthguards are widely recommended as an essential piece of protective equipment for football players. ${ }^{12}$ A number of retrospective studies investigating mouthguards have shown a general trend towards a reduction in the incidence and severity of orofacial injuries among mouthguard users. ${ }^{3-5}$ The scientific evidence for their protection against head injuries is limited, although this has never been tested in a prospective study. ${ }^{6}$ Although not compulsory in Australian rules football, mouthguard use is widespread at the higher levels of competitive play. ${ }^{7}$ At the community level, usage is less at approximately $60-70 \%$ of players during games and almost non-existent during training. ${ }^{3}$

Most mouthguards used by players are the over the counter "boil and bite" type and are considered to have limited effectiveness in injury prevention compared with custom made mouthguards. ${ }^{2}{ }^{8}$ Despite the widespread support given to mouthguards in football, controlled trials of mouthguards in the football field have not been undertaken to date. ${ }^{28}$

Ethylene vinyl acetate (EVA) custom mouthguards have been shown in laboratory tests to offer superior protection to the teeth than a boil and bite design. ${ }^{9}$ However, within custom EVA mouthguards, variations in thickness, density, and coverage influence the level of protection provided, ${ }^{9}$ - for example, $5 \mathrm{~mm}$ thick EVA offered the greatest protection and extending the guard to behind the first molar offered further benefits. Westerman et al observed no improvements in the impact energy attenuation performance of $4 \mathrm{~mm}$ thick EVA mouthguards with the introduction of gas cells in the foam. ${ }^{10}$ A finite element study of mouthguard performance found that mouthguard stiffness is very important to provide load distribution and protect the teeth, leading to the conclusion that either sports specific mouthguards are required, or that composite materials are required that both attenuate energy and distribute load. ${ }^{11}$

The aim of this study was to determine the extent to which custom made mouthguards are effective for the prevention of head/orofacial $(\mathrm{H} / \mathrm{O})$ injuries in Australian rules football players. It was hypothesized that custom made mouthguards would reduce the rate of $\mathrm{H} / \mathrm{O}$ injuries, relative to usual mouthguard behaviors.

\section{METHODS}

\section{Design}

A group randomized controlled trial (RCT) design was used because players belong to football teams and interventions need to be delivered at the team level, whereas the injury impacts are measured at the player level. Initially, this study was designed as a four arm factorial trial to also assess the effectiveness of headgear in these players (headgear, mouthguards, headgear+mouthguard, control). However, so few players wore headgear ${ }^{12}$ that it was not possible to assess its effectiveness and so the study design was collapsed to a two

Abbreviations: EVA, ethylene vinyl acetate; $\mathrm{H} / \mathrm{O}$, head/orofacial; IRR, incidence rate ratio; PDC, primary data collector; RCT, randomized controlled trial. 
Table 1 The Australian football injury prevention project design and numbers of recruited teams and players

\begin{tabular}{|c|c|c|}
\hline Intervention arm & $\begin{array}{l}\text { Control (usual } \\
\text { behaviours) (C) }\end{array}$ & $\begin{array}{l}\text { Custom made } \\
\text { mouthguard (MG) }\end{array}$ \\
\hline No of teams randomized & 15 & 12 \\
\hline No of teams participating & 11 & 12 \\
\hline No of randomization units* & $* 5$ & 8 \\
\hline \multicolumn{3}{|c|}{ Division of team } \\
\hline$\|$ & 1 & 3 \\
\hline III & 1 & 2 \\
\hline IV & 3 & 3 \\
\hline \multicolumn{3}{|l|}{ Teams } \\
\hline Seniors (firsts or reserves) & 5) 4 & 4 \\
\hline Juniors (U16 or U18) & 1 & 4 \\
\hline \multicolumn{3}{|l|}{ Players } \\
\hline No eligible players & 238 & 224 \\
\hline No recruited players & 111 & 190 \\
\hline Players response rate & $47 \%$ & $85 \%$ \\
\hline \multicolumn{3}{|l|}{$\begin{array}{l}\text { Total exposure } \\
\text { (1000 hours) }\end{array}$} \\
\hline Games & 134.4 & 235.3 \\
\hline Training & 166.6 & 336.9 \\
\hline \multicolumn{3}{|l|}{$\begin{array}{l}\text { Average exposure per } \\
\text { player per week (minutes) }\end{array}$} \\
\hline Games & 106 (range 80-120) & 105 (range $80-120$ ) \\
\hline Training & 141 (range 60-180) & 153 (range 60-180) \\
\hline
\end{tabular}

arm factorial trial. Table 1 summarizes the two arms of the study, the allocation of randomization units to study arms, and player recruitment rates.

\section{Intervention}

Teams of players were randomly allocated to one of two mouthguard wearing behaviors: MG, custom made mouthguards and C, control (usual mouthguard behaviors).

Players assigned to the MG arm were provided with a trilaminate mouthguard custom fitted by a dental technician

Table 2 Baseline characteristics of players recruited to each study arm

\begin{tabular}{|c|c|c|}
\hline Variable & $C(n=111)$ & MG $(n=190)$ \\
\hline \multicolumn{3}{|l|}{ Division, n (\%) } \\
\hline$\|$ & $43(39 \%)$ & $51(27 \%)$ \\
\hline III & $11(10 \%)$ & $74(39 \%)$ \\
\hline IV & $57(51 \%)$ & $65(34 \%)$ \\
\hline \multicolumn{3}{|l|}{ Team, n (\%) } \\
\hline Seniors & $76(69 \%)$ & $127(67 \%)$ \\
\hline Under 18 & $11(10 \%)$ & $44(23 \%)$ \\
\hline Under 16 & $24(22 \%)$ & $19(10 \%)$ \\
\hline \multicolumn{3}{|l|}{ Age (mean (SD)) } \\
\hline Seniors & $26(4.6)$ & $24(5.1)$ \\
\hline Juniors & $16(1.1)$ & $17(1.2)$ \\
\hline \multicolumn{3}{|l|}{ BMI (mean (SD)) } \\
\hline Seniors & $27(3.1)$ & $26(2.7)$ \\
\hline Juniors & $23(3.4)$ & $23(3.0)$ \\
\hline \multicolumn{3}{|c|}{ Previous head/orofacial or neck injury, $n(\%)$} \\
\hline Yes & $17(15 \%)$ & $31(16 \%)$ \\
\hline No & $94(85 \%)$ & $159(84 \%)$ \\
\hline \multicolumn{3}{|c|}{ Wore mouthguard in previous season, $\mathrm{n}(\%)$} \\
\hline Yes & $89(79 \%)$ & $176(90 \%)$ \\
\hline No & $22(21 \%)$ & $14(10 \%)$ \\
\hline $\begin{array}{l}\text { Pre-season mouthguard risk taking } \\
\text { behavior score (mean (SD)) }\end{array}$ & $19.1(2.5)$ & $19.5(2.6)$ \\
\hline \multicolumn{3}{|l|}{ Perception of head injury risk } \\
\hline None/low & $67(61 \%)$ & $97(52 \%)$ \\
\hline Moderate & $37(34 \%)$ & $72(38 \%)$ \\
\hline High & $6(5 \%)$ & $19(10 \%)$ \\
\hline \multicolumn{3}{|l|}{ Perception of mouth/dental injury risk } \\
\hline None/low & $22(21 \%)$ & $25(13 \%)$ \\
\hline Moderate & $53(50 \%)$ & $94(50 \%)$ \\
\hline High & $32(30 \%)$ & $68(36 \%)$ \\
\hline
\end{tabular}

at no cost. They were made from polyvinyl-acetate-polyethylene and chosen because formal materials testing demonstrated their protective efficacy in laboratory studies suggesting that they provided the best protection for teeth compared with other available mouthguards. ${ }^{13}$ The dental technicians took moulds of each player's top and bottom teeth so that the mouthguard could be moulded exactly to fit their specific dentition.

Players were requested to wear their allocated protective equipement for all training sessions and competitive games during the 2001 playing season. For ethical reasons it was not possible to restrict mouthguard use in control players and some may have chosen to wear a custom fitted mouthguard of a different type. Mouthguard use, whether of allocated equipment or self provided, was monitored for every player (as described below).

\section{Randomization and recruitment}

Teams of players from clubs from the largest district Australian rules football competition in Australia were invited to participate. All 40 clubs were invited to participate and nine did so. Although involved in competitive play, no player was an elite or high level player.

Randomization to study arms occurred after clubs/teams agreed to participate in the study. The randomization units were teams of players from the under 16 years, under 18 years, and open (seniors and reserves) competition levels. Seniors and reserves teams were randomized together because players often swap between these teams within a club during the season. Randomization was stratified by level of competition to balance age across the study arms.

After team study arm allocation, all players were given information about the study, the protective equipment they would be required to wear (if any) and details of the recruitment and study procedures. Player recruitment was undertaken on site over two club training sessions during the pre-season period. Each recruited player was given a plain language statement and completed an informed consent form. The study was approved by the Deakin University Ethics Committee. Data collection procedures were only completed for players who consented to be part of the study. Non-responder surveys were undertaken to ascertain why players and clubs did not volunteer for this study. ${ }^{14} 15$

\section{Data collection}

Players completed a piloted baseline questionnaire ${ }^{16}$ on their demographic profile, football playing history, previous football injury history, general attitudes towards protective equipment, self reported usual behaviors in relation to protective equipment, and risk perceptions. Full details of this study component have been published elsewhere. ${ }^{17}$

All field work procedures were standardized. A primary data collector (PDC) was nominated from each team to undertake the on site data collection during each game and training session. All PDCs received formal training in the data collection procedures and were given a procedures manual including the data collection forms for recording exposure, compliance, and injuries, and full details of the coding and classification schemes. The accuracy of the PDCs' activities was assessed by an independent auditing process and found to be highly reliable. ${ }^{18}$

The PDCs recorded injured player details for each game and training session including a description of all injuries sustained and the injury circumstances. Information about injury outcomes (that is, time away from sport and amount of treatment received) was also collected. The injury details have been published elsewhere. ${ }^{19}{ }^{20}$ The data were obtained from the PDCs on a regular basis throughout the playing season. For each player, the amount of time (in hours) spent 
playing football (both competition and training) over each week of the trial was also recorded. The PDCs also reliably recorded whether or not players actually used their allocated protective equipement each week. ${ }^{18}$

Head/orofacial injuries were defined as any injury, irrespective of severity, to the forehead, scalp, ears, skull, brain, face, jaw, or dentition.

\section{Statistical analysis}

Analyses were performed with STATA ${ }^{21}$ and performed on an intention to treat basis, with the control arm as the reference in the calculation of rate ratios. Baseline player characteristics were examined for equivalence in injury risk at baseline across the study arms.

Injury rates were calculated as the number of injuries divided by time exposed (at training or games) and expressed per 1000 person hours of playing time. Incidence rate ratios (IRR) were obtained from Poisson regression models, with a player's number of injuries in a game or training session as the outcome variable and exposure time as a log offset term, together with risk factors in the linear predictor part of the model. The factors considered for adjustment in these models are shown in table 2 . Incidence rate ratios for time-varying risk factors were able to be calculated because the data were split into individual units of exposure (that is, training sessions and games for each player in each round). Robust standard errors were calculated using the information sandwich formula to account for clustering of players in the original units of randomization. ${ }^{21}$

\section{RESULTS}

Table 1 describes the composition of the study sample and the randomization units. After randomization, four teams dropped out of the study for reasons unrelated to the study arm allocation: not fielding a team for the 2001 playing season; not being able to complete the required study procedures of injury surveillance and exposure recording; or unable to arrange a time for player recruitment.

There was some imbalance of teams between the two study arms-for example, a greater proportion of the control teams were seniors compared with the MG group; however the balance of players between study arms was reasonable (table 2). The player recruitment rate was higher in the MG arm and hence there was more total exposure in this arm; however average game time and training exposure per player per week was similar in the two arms. Apart from the division of play, there were no major differences between study arms.

Irrespective of the study arm, the vast majority of players wore mouthguards during games, indicating that most control players still chose to wear mouthguards. In contrast, few players wore mouthguards during every training session.

The $\mathrm{H} / \mathrm{O}$ injury rate was 2.7 injuries per 1000 hours of participation (95\% CI 1.6 to 4.5 ). The most common $\mathrm{H} / \mathrm{O}$ injury was a facial laceration (38\% of all $\mathrm{H} / \mathrm{O}$ injuries) and these were 1.4 times more common in control players than MG players. Concussions (19\% of all H/O injuries) were distributed roughly equally across study arms. The only player from the MG arm to receive a dental injury was not wearing their mouthguard at the time of injury.

The overall injury rate was 12.8 (95\% CI 7.1 to 23.1 ) injuries per 1000 participation hours. There was a higher rate of $\mathrm{H} / \mathrm{O}$ injury in the control arm than in the MG arm, when training and game exposures were combined: 4.4 (95\% CI 2.2 to 8.9 ) injuries/1000 exposure hours versus 1.8 (95\% CI 1.1 to 2.9) injuries/1000 exposure hours, respectively. The H/O injury rate was significantly higher during games than training sessions both overall (5.8 (95\% CI 3.5 to 9.6) $v 0.4$ ( $95 \%$ CI 0.1 to 1.4 ), respectively) and in both study armscontrols (8.9 (95\% CI 4.3 to 18.6$) \vee 0.7$ (95\% CI 0.1 to 3.1$)$,

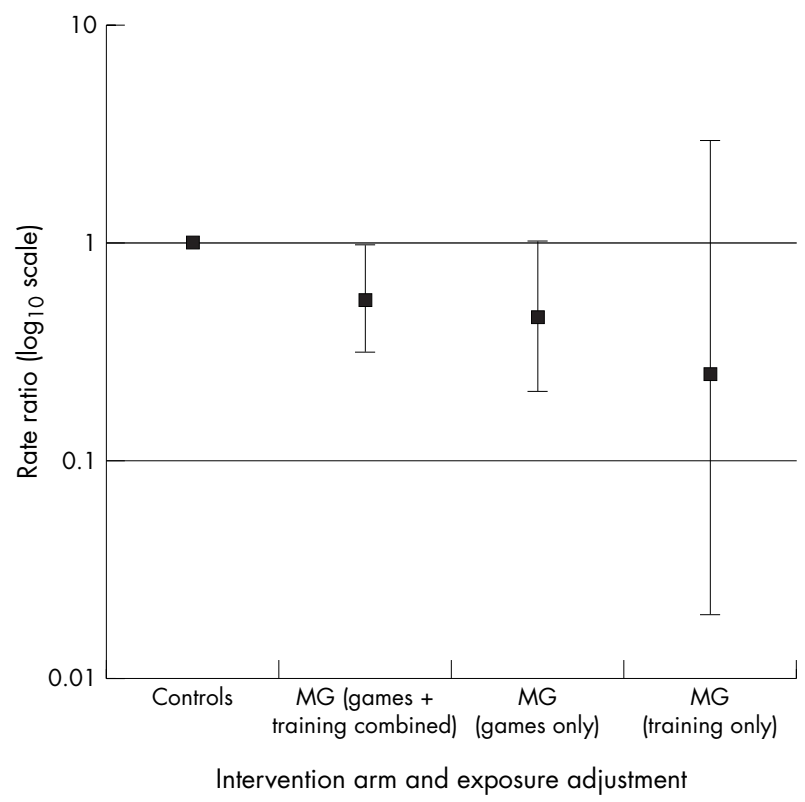

Figure 1 Incidence rate ratios comparing adjusted head/orofacial injury rates in the mouthguard arm, relative to controls $(p=0.06$ for mouthguards $v$ controls during games and $p=0.04$ for mouthguards $v$ controls during games and training combined).

respectively); MG arm (4.1 (95\% CI 2.5 to 6.7$) v 0.2$ (95\% CI 0.0 to 1.2 ), respectively).

Figure 1 shows the study arm H/O injury rate comparisons, after adjusting for division of play and age group-two factors showing an association with injury and imbalance between study arms (table 2). There was some evidence of a protective effect of mouthguards during games and training combined (unadjusted IRR $=0.41$; adjusted IRR $=0.56 ; 95 \%$ CI 0.32 to $0.97: p=0.04)$. When only game exposure was considered, the MG arm had a reduced rate of $\mathrm{H} / \mathrm{O}$ injuries relative to controls (unadjusted IRR $=0.46$; adjusted IRR $=0.60 ; 95 \%$ CI 0.35 to $1.02 ; \mathrm{p}=0.06$ ).

\section{DISCUSSION}

Given the relatively high level of participation in Australian rules football by Australian males and the predominance of associated injury presentations at medical services, this sport is a high priority for injury prevention research. ${ }^{8}$ Although preventive measures have been adopted to varying degrees, the current lack of an evidence base about their effectiveness limits the safety advice that can be given to football participants, their parents, coaches, and sports administrators. ${ }^{8}$ This study is the first RCT of the effectiveness of any injury prevention measure in community Australian rules football and one of only a very few in any level of football participation.

Although the protective effect of mouthguards against head injury remains theoretical ${ }^{22}$ with only anecdotal ${ }^{23}$ or limited clinical evidence ${ }^{24} 25$ to suggest a beneficial effect, it was nevertheless important to test this premise of mouthguard efficacy in a controlled study. It can be intuitively appreciated that mouthguards are likely to offer a more protective effect in relation to orofacial injuries. However, at this level of football such injuries are uncommon and a controlled study would have needed to be significantly larger in order to have adequate power to detect a benefit if present. Given the budgetary and logistic constraints of this study, it was not considered feasible to test these hypotheses separately. 
Our study has demonstrated that custom fitted mouthguards provide significant protection against H/O injuries, relative to usual mouthguard behaviors, during combined games and training exposures. Wisniewski et al found no difference in the rate of concussion between players wearing boil and bite mouthguards and custom made mouthguards in National Collegiate American Football. ${ }^{26}$ Mouthguards only provide impact energy attenuation during impacts to the mouth, and therefore have a very limited function in preventing concussion.

Comparing the player recruitment rate in the two study arms suggests that the provision of custom made mouthguards was a significant incentive for players to participate. However, although players adopted mouthguards for the game situation, fewer did so during training, even though there is still a risk of $\mathrm{H} / \mathrm{O}$ injury. Even with optimally designed protective equipement, unless players choose to use it, it will not be an effective form of protection. There is no necessity for players to wear mouthguards unless engaged in training that involves potential contact. But wearing protective equipment during training is important as it provides an opportunity for players to develop an awareness of its limitations and usability issues-for example, comfort and communication.

The magnitude of the protective effect demonstrated for the protective equipement tested in this trial across training and game sessions is most likely to be related to (a) the fact that players did not wear their allocated protective equipment all of the time and (b) the study being underpowered. Player recruitment was affected by the ethical and practical requirement of informing players of what they would be required to do before they signed up for this study.

Another problem with the study was the nature of the control arm. For ethical reasons, we could not prohibit control players from wearing their usual mouthguards, if any. This has led to a likely dilution of the differences between the two study arms, as the control arm was not a true control. Thus this study did not test mouthguard wearers against non-mouthguard wearers. Rather it could only compare players who wore the supplied mouthguard to those who wore their own mouthguards. However, baseline assessment and previous published research indicated that players generally do not choose to use custom fitted mouthguards. ${ }^{7}$

This study has a number of significant strengths. Firstly, it adopted an RCT methodology, which is regarded as providing the highest level of evidence for the effectiveness of an intervention. A group randomized design was used because it replicated the setting of application of the interventions in the real world. Thus the study provides a model for future sports injury research. Secondly, the intervention had previously undergone rigorous lab based testing and its impact properties were well known. Thirdly, the study adopted a rigorous and highly reliable data collection methodology. ${ }^{18}$

Block allocation of junior (U16 and U18) and senior (seniors and reserves) teams to study arms was used to balance for most factors. However, this balance did not extend to division of play. Conventionally, better skilled players participate in a higher level of competition. Division IV is a lower level competition than Division III or II. As a higher proportion of control teams were Division IV players, we adjusted for this factor as well as age group in our comparisons. Some of the injury rate difference is explained by these factors, although a protective effect of borderline statistical significance remained.

This first randomized controlled trial in community level Australian football players has shown that they do not always choose to wear protective equipment. However, custom fitted mouthguards seem to be protective against $\mathrm{H} / \mathrm{O}$ injuries,

\section{Key points}

- This is the first randomized controlled trail of the effectiveness of protective equipment in community level Australian football, and one of a few worldwide in any sport.

- Players who were allocated to the custom fitted mouthguard study arm had a significantly lower rate of head and orofacial injuries than all other players.

- There are significant challenges in conducting randomized controlled trials in sporting populations.

relative to usual mouthguard behaviors. It is therefore recommended that Australian football players should wear custom fitted mouthguards, rather than the boil and bite type or no mouthguards.

\section{Authors' affiliations}

C Finch, NSW Injury Risk Management Research Centre, University of New South Wales, Sydney, NSW, Australia

R Braham, R Wolfe, Department of Epidemiology and Preventive Medicine, Monash University, Victoria, Australia

A McIntosh, School of Safety Science, University of New South Wales, Sydney, NSW, Australia

P McCrory, Centre for Health, Exercise and Sports Medicine \& the Brain Research Institute, University of Melbourne, Melbourne, Australia

$R$ Braham is now at the School of Human Movement and Exercise Science, University of Western Australia

\section{REFERENCES}

1 National Health and Medical Research Council. Football injuries to the head and neck, 1995.

2 Chalmers DJ. Mouthguards. Protection for the mouth in rugby union. Sports Med 1998;25:339-49.

3 Jolly K-A, Messer LB, Manton D. Promotion of mouthguards among amateur football players in Victoria. Aust N Z J Public Health 1996;20:630-9.

4 Scott J, Burke F, Watts D. A review of dental injuries and the use of mouthguards in contact team sports. Dental Trauma 1994;176:310-14

5 Jennings DC. Injuries sustained by users and non-users of gum shields in local rugby union. Br J Sports Med 1990;24:159-65.

6 McCrory P. Do mouthguards prevent concussion? Br J Sports Med 2001;35:81-2.

7 Banky J, McCrory P. Mouthguard use in Australian Football. J Sci Med Sport 1999;2:20-9.

8 Gabbe B, Finch C. Injury countermeasures in Australian Football. J Sci Med Sport 2000;3:31-40.

9 Greasley A, Imlach G, Karet B. Application of a standard test to the in vitro performance of mouthguards. BrJ Sports Med 1998;32:17-19.

10 Westerman B, Stringfellow P, Eccleston J, et al. Effect of ethylene vinyl acetate (EVA) closed cell foam on transmitted forces in mouthguard material. Br J Sports Med 2002;36:205-8.

11 Cummins N, Spears I. The effect of mouthguard design on stresses in the toothbone complex. Med Sci Sports Exerc 2002;34:942-7.

12 Braham RA, Finch CF. Do community football players wear allocated protective equipment? Results from a randomised controlled trial. J Sci Med Sport 2004;7:216-20

13 Westerman B, Stringfellow PM, Eccleston JA. An improved mouthguard material. Aust Dental J 1997:42:189-91.

14 Braham R, Finch C, McCrory P. Non-participation in sports injury research: why football players contribute to research projects. Br J Sports Med 2004;38:238-9.

15 Zazryn T, Braham R, Finch C. Clubs volunteering for sports injury prevention research - is there any selection bias? Inj Control Saf Promot 2004;1 1:67-9.

16 Finch C, Mclntosh A, McCrory P, et al. A pilot study of the attitudes of Australian rules footballers towards protective headgear. J Sci Med Sport 2003;6:505-11.

17 Braham RA, Finch CF, Mclntosh A, et al. Community football players' attitudes towards protective equipment-a pre-season measure. Br J Sports Med 2004;38:426-30

18 Braham RA, Finch CF. The reliability of team-based primary data collectors for the collection of exposure and protective equipment use data in community sport. Br J Sports Med 2004;38:e15.

19 Braham R, Finch CF, Mclntosh A, et al. Community level Australian Football: a profile of injuries. J Sci Med Sport 2004;7:60-8. 
20 Braham R, Finch C, McCrory P. The incidence of head/neck/or facial injuries in non-elite Australian football. J Sci Med Sport 2004;7:451-3.

21 StataCorp. Stata Statistical Software: Release 7.0. College Station, TX: Stata Corporation, 2001

22 Stenger. Mouthguards: protection against shock to head, neck and teeth. J Am Dent Assoc 1964;19:273-81.

23 Hickey J. The relation of mouth protectors to cranial pressure and deformation. J Am Dent Assoc 1967;74:735-40.
24 Chapman PJ, Nasser BP. Attitudes to mouthguards and prevalence of orofacial injuries in four teams competing at the second Rugby World Cup. Br J Sports Med 1993;27:197-9.

25 Chapman P. Concussion in contact sports and importance of mouthguards in protection. Aust J Sci Med Sport 1985:17:23-7.

26 Wisniewski J, Guskiewicz K, Trope M, et al. Incidence of cerebral concussions associated with type of mouthguard used in college football. Dent Traumatol 2004;20: 143-9.

\section{LACUNAE}

\section{State "protecting" traffic information}

$\mathrm{H}$

awaii's Transportation Department spends millions of dollars each year studying traffic safety, but the agency does not share the findings with the public or other government agencies. Hawaii is one of a few US states that withhold the information to protect against lawsuits claiming the state moved too slowly to correct known traffic hazards. Transportation officials say the threat of multi-million dollar settlements justifies withholding the information, and they would rather use the money for safety improvement projects. "I'd like to make the state a model for information-sharing like other places, where they don't have to worry about having that information thrown back into their faces by the attorneys," said Alvin Takeshita, head of the state DoT's Traffic Branch. Under state and federal law, state traffic data are inadmissible in civil cases, but Takeshita said attorneys get around the law by introducing county police reports with the same information. Other states have opted to release the data after redacting personal information about those involved in crashes. Some states have tried to make the data as accessible as possible by posting the information on public websites, so that drivers and pedestrians can see which locations have the most crashes. Critics of the state's policy say the public needs to see the state data to determine whether state and county transportation officials are making good decisions about which crash locations to improve or repair, and to make informed decisions about where and when to travel.

Reported in CDC Public Health Law News. Contributed by Ian Scott.

\section{Well-fed goldfish}

Russian woman's fingers were badly bitten when she tried to clean her son's fish
tank, unaware the fish were piranhas. She told doctors she thought they were well-
fed goldfish. The Age (Melbourne), May 2005. Contributed by Ian Scott. 